\title{
Conclusiones de las VIII Jornadas Nacionales y IV Internacionales de Educación en Fisioterapia
}

\author{
Conclusions of the VIII National and IV International Meetings of Education in \\ Physiotherapy
}

\author{
J. Seco Calvo, S. Souto Camba
}

Los pasados días 22 al 24 de abril se celebraron en el Campus Universitario de Ponferrada las VIII Jornadas Nacionales y IV Internacionales de Educación en Fisioterapia y las VIII Jornadas Interuniversitarias del Área de Conocimiento Fisioterapia, organizadas por la Asociación Española de Fisioterapeutas, a través de su sección de educación, y la Escuela Universitaria de Ciencias de la Salud y Departamento de Enfermería y Fisioterapia de la Universidad de León, bajo el lema «Investigación clínica, convergencia y calidad».

Su proyección internacional quedó patente a través de la presencia de congresistas de Argentina, Estados Unidos, Suecia, Holanda, México y Portugal. De España, asistieron docentes y alumnos universitarios de todas las comunidades autónomas hasta reunir un total de 221 congresistas, que han aportado los resultados de sus investigaciones sumando un total de 87 comunicaciones aceptadas (73 en cartel y 14 orales). Recordando palabras del Dr. Rebollo en la ceremonia de clausura, queda una gran satisfacción por el nivel y calidad mostrados en los trabajos presentados que están recogidos en un libro de actas publicado y editado con ISBN 978-84-613-9646-7, cuya propiedad intelectual ostentan conjuntamente ambas instituciones organizadoras.

La Jornada Interuniveritaria, cuya dinámica es en base a grupos de trabajo («flexibilidad docente», «universidad 3.0» $\mathrm{y}$ «calidad») las conclusiones fueron:

a. «Flexibilidad docente»:

- El desarrollo de los planes de estudio adaptados al EEES es muy rígido, pero a la vez precisa de una enorme variabilidad y flexibilidad en la docencia.

- La innovación docente y el esfuerzo que requiere están poco reconocidos formalmente en la hoja de servicios del profesor. Se solicita su reconocimiento curricular.

- Se han observado disparidades entre las distintas universidades en la distribución de grupos, tutorías, prácticas de laboratorio, etc., que sería conveniente unificar por el bien de la formación de calidad del alumno.

b. «Universidad 3.0.», las conclusiones, fueron realizadas siguiendo el espíritu de trabajo de la «nube»:

- A nivel docente, la creación de «nubes» o discos duros virtuales nos ofrece una herramienta con múltiples usos adaptada a los nuevos requisitos a nivel metodológico de las titulaciones de grado, tanto para la docencia presencial como para la no presencial.

- A nivel científico, supone una revolución metodológica, pasando del antiguo proceso de entrevista y/o encuestas individuo a individuo de modo presencial, pudiendo realizarlo con esta herramienta de modo no presencial, multiplicándose la obtención de resultados, además de la capacidad de este sistema de procesar los datos obtenidos de modo automático.

- Facilitación del proceso de recogida de datos en proyectos de investigación y de su análisis posterior.

- Permite, además, el control y la comunicación entre los diferentes centros asistenciales y de sus tutores y/o profesores asociados de prácticas clínicas, así como el intercambio de documentos realizado entre ello y los alumnos. 
- Es una base de datos, un método de enseñanza/aprendizaje y un medio de comunicación, todo en uno, que adapta de modo óptimo el procedimiento educativo a los nuevos tiempos y requerimientos, siendo de gran aplicabilidad en el ámbito de la docencia e investigación dentro del área de fisioterapia.

- El trabajo en «nube» facilita las tareas docentes en el EEES.

\section{c. $\ll$ Calidad»:}

- Se ha de concienciar a todos los componentes del ámbito universitario (profesorado, alumnos, PAS, equipos rectorales...) de cuáles son los componentes del criterio 9, calidad.

- Se ha de conseguir el correcto funcionamiento de los mecanismos del seguimiento del control de calidad.

- El proceso de verificación está ligado a la calidad, la autorización ligada a la financiación. Podría darse la situación de tener un título autorizado pero no verificado.

- Los indicadores de resultados necesarios para obtener la acreditación solo serán conseguidos si existe una financiación adecuada.

- Es necesario generar y desarrollar unos indicadores que nos lleven a la calidad de la enseñanza.

- Se propone la mejora en la valoración del trabajo en todos los ámbitos de la calidad.

- Existen responsables en las comunidades autónomas y en el consejo interuniversitario y dentro de las pocas universidades hay comisiones de calidad, entendemos que estas comisiones de seguimiento de los títulos deben ser mecanismos de referencia para la evaluación continuada de la titulación.

Se presentan a continuación las conclusiones de las 3 mesas de debate de las VIII Jornadas Nacionales y IV Internacionales de Educación en Fisioterapia.

\section{Conclusiones de la primera 1. ${ }^{\mathrm{a}}$ Mesa: «Capacidad facultativa en fisioterapia»}

La fisioterapia es un término que posee, al menos, 3 significados. Se corresponde con un campo del saber y constituye una ciencia, identifica un título académico y denomina una profesión. La Confederación Mundial por la Fisioterapia (WCPT) ya recoge en su documento «Description of Physical Therapy», aprobado en el año 1999 y revisado en el 2007, estos 3 elementos al reconocer que la fisioterapia debe estar basada en el desarrollo de su área de conocimiento, la educación a nivel académico y la responsabilidad en el ejercicio autónomo de la profesión con el objetivo de prestar el mejor servicio posible a los individuos y a la sociedad.

De forma consecuente, el pleno desarrollo de la fisioterapia pasa por su pleno desarrollo a nivel académico, por la defensa y mantenimiento de su ejercicio autónomo, y el establecimiento de sus propias bases de conocimiento en las que sustentar la práctica profesional a través de la investigación.

En el transcurso de las diferentes intervenciones desarrolladas por los ponentes del ámbito internacional, los profesores David Alan Arnall, Director del Departamento de Fisioterapia de la East Tennesse University de EE.UU., la profesora Raija Tyni-Lenne, catedrática de Instituto Karolinska de Suecia, y el profesor Henri Kiers, representante de la Real Sociedad Holandesa de Fisioterapia, quedó patente la necesidad de que la fisioterapia española se adecue a los paradigmas y normas en los que se mueven estos países, como son el acceso directo a fisioterapia, la existencia de jerarquización clínica de los fisioterapeutas y el reconocimiento de las especialidades en el ámbito clínico.

El Dr. Ramón Fernández Cervantes, director de la Escuela Universitaria de Fisioterapia de A Coruña, puso de manifiesto que la situación actual en España, derivada del proceso de adaptación al Espacio Europeo de Educación Superior, supone una etapa de crisis que debe ser adecuadamente gestionada por la fisioterapia española para alcanzar su pleno desarrollo profesional, por ello, propuso que la práctica de la fisioterapia amplíe su campo competencial para ejercer competencias que ya están siendo desempeñadas por países tan avanzados en ciencia y conocimiento como EE.UU., Australia, Reino Unido, Suecia, etc.

Su propuesta, que fue avalada y aprobada por el pleno de asistentes a las Jornadas, se concertó en 6 objetivos fundamentales a conseguir:

1. Acceso directo de los pacientes a la consulta de fisioterapia.

2. Capacidad de prescribir, a nivel de ejercicio privado y público, no solo el programa de intervención terapéutica, sino también cualquier producto sanitario (órtesis, prótesis, bastones, etc.) necesario para el tratamiento de fisioterapia.

3. Capacidad de emitir diagnósticos relacionados con las alteraciones de la función a nivel corporal y de persona. 
4. Capacidad para solicitar pruebas diagnósticas básicas relacionadas con la elaboración de los diagnósticos propios, como pueden ser: radiografías, ecografías, electromiografías, analíticas de sangre y orina.

5. Capacidad de derivar para interconsulta con otros profesionales de la salud.

6. Reconocimiento de las especialidades en el Sistema Nacional de Salud.

\section{Conclusiones de la 2. ${ }^{a}$ Mesa: «el reto del cuarto año clínico»}

Se ha resaltado la importancia de la transversalidad de los contenidos en la adquisición de las competencias dentro del proceso de enseñanza/aprendizaje, así como la realización de evaluaciones conjuntas.

Es fundamental para la formación clínica la coordinación de los contenidos teóricos y prácticos, debiendo enfocarse el proceso de enseñanza/aprendizaje al razonamiento clínico, siendo para ello fundamental contar con profesorado formado y realizar dicha formación en centros conveniados.

Una de las estrategias para la formación clínica es que las universidades cuenten entre sus recursos propios con una clínica universitaria de fisioterapia, sirviendo además para mejorar e incrementar la investigación en fisioterapia.

Atendiendo al último ítem del lema («calidad»), se ha de resaltar que no se debería poder impartir la titulación de fisioterapia en universidad alguna sin plena y total garantía de calidad, particularmente en la formación clínica, lo que significa que las instituciones (universidades y consejerías de educación y de sanidad de las distintas comunidades autónomas), como responsables ante la sociedad de la calidad formativa y de la competencia profesional de los futuros fisioterapeutas, deben realizar un esfuerzo inversor para dotar de profesorado, y particularmente de profesorado clínico, para que los alumnos sean formados debidamente.

Se acuerda solicitar a la Administración (Dirección General de Sanidad y al Ministerio de Educación que exija a las universidades tanto públicas como privadas que la formación clínica se realice en centros conveniados y contando con las figuras de profesorado adecuadas. Así mismo hacer llegar esta solicitud a la Conferencia Nacional de Directores de Escuelas Universitarias de Fisioterapia, al Consejo General de Colegios Oficiales de Fisioterapeutas y a los distintos colegios oficiales de fisioterapeutas.

\section{Conclusiones de la 3. ${ }^{a}$ Mesa: «vinculación entre fisioterapia académica y la investigación clínica»}

Para el pleno desarrollo de una disciplina en el ámbito científico, es imprescindible que los profesionales que se encarguen de la tarea investigadora estén formados específicamente en su propio campo de conocimiento y que conformen sus propias líneas de investigación.

Los centros, y los departamentos de fisioterapia implicados, deben poder ofrecer con plena garantía y total autonomía sus programas de máster y doctorado, y debe ser el profesorado del área de conocimiento «fisioterapia» quien los coordine. Las autoridades académicas, legislativas y ministeriales tienen la obligación y la responsabilidad de ser los garantes de este proceso.

En relación con la evaluación de los tramos de la actividad investigadora, en la actualidad, con la nueva ley orgánica de universidades, los sexenios se han convertido, además, en un indicador de calidad y son imprescindibles para formar parte de las comisiones de habilitación y de las comisiones de las universidades que juzgan el concurso de acceso. Este cambio significa que un complemento meramente económico ha sido elevado a un indicador de excelencia académica. Por ello, y a propuesta de la Dra. M. Teresa Labajos Manzanares, Presidenta de la Conferencia Estatal de Directores de Escuela de Fisioterapia, se acuerda solicitar a la Comisión Nacional de Evaluación de la Actividad Investigadora (CNEAI):

1. En relación a las futuras evaluaciones en todas las áreas y comisiones:

- Que se rediseñen los campos científicos, evitándose los formados a partir de la adición de áreas de conocimiento muy dispares.

- Que existan especialistas de todas las áreas de conocimiento en los comités asesores para que se produzca un acercamiento más real a los méritos de los trabajos que han de juzgar.

- Que en el nombramiento de los expertos no influyan criterios que no sean estrictamente académicos.

- Que todo el proceso y los resultados sean transparentes y justificados.

- Que se valoren los trabajos en equipo evitando las disfunciones que se observan en la actualidad. 
- Que, en la revisión o impugnación, se admita informe de perito de parte o, al menos, proposición de terna de expertos en el área de conocimiento y que resuelva otro comité asesor distinto.

2. En relación a las áreas discriminadas («fisioterapia» en los 13 años anteriores):

- Que los trabajos y aportaciones de quienes no han conseguido sexenios y lo soliciten, se sometan a una nueva evaluación aunque no genere efectos económicos retroactivos.

- Que en esa revisión, los sexenios anteriores deben ser evaluados teniendo en cuenta en cada caso el nivel científico existente en nuestro país para cada periodo que se somete a evaluación.

- Que se modifique la Resolución de 18 de noviembre de 2009, de la Presidencia de la Comisión Nacional Evaluadora de la Actividad Investigadora, por la que se establecen los criterios específicos en cada uno de los campos de evaluación (BOE n. ${ }^{\circ} 289$ de martes 1 de diciembre de 2009) y se permita al profesorado del área de conocimiento «fisioterapia»ser evaluado no solo por el Campo 4, «ciencias biomédicas», sino también por el Campo 7, «ciencias sociales, políticas, del comportamiento y de la educación».

3. A propuesta de la Dra. Antonia Gómez Conesa, Catedrática de Fisioterapia y Presidenta de la Asociación Española de Fisioterapeutas, se acuerda elevar a las autoridades correspondientes, que la investigación clínica en el ámbito de Fisioterapia, requiere de los sistemas sanitarios:

- El desarrollo de nuevas políticas de RRHH en los sistemas sanitarios, que reconozcan la autonomía profesional investigadora de los profesionales fisioterapeutas capacitados para la misma.

- La creación de becas para investigación en Fisioterapia, que ayude realizar estudios de posgrado y tesis doctorales en el ámbito clínico.

- El establecimiento de conciertos con la Universidad para el desarrollo de proyectos conjuntos entre los investigadores académicos y los clínicos.

Nuestro afecto y agradecimiento a todos los que han contribuido con su ilusión y dedicación a la consecución exitosa de las Jornadas. 